\title{
VALIDACIÓN DEL ÍNDICE DE ESTRÉS HÍDRICO DE CULTIVO (CWSI) MEDIANTE TERMOGRAFÍA INFRAROJA Y SU INCIDENCIA EN RENDIMIENTO Y CALIDAD EN MANZANAS 'ROYAL GALA'
}

\author{
VALIDATION OF CROP WATER STRESS INDEX (CWSI) BY INFRARED \\ THERMOGRAPHY AND ITS IMPACT ON FRUIT YIELD AND QUALITY OF \\ 'ROYAL GALA' APPLES
}

\author{
Celerino Quezada ${ }^{1 *}$, Richard Bastias $^{1}$, Rodrigo Quintana $^{2}$, Raimundo Arancibia $^{1}$, Alejandro Solís ${ }^{1}$ \\ ${ }^{1}$ Facultad de Agronomía, Universidad de Concepción, Casilla 537, Chillán, Chile. \\ ${ }^{2}$ Instituto de Investigaciones Agropecuarias, Centro Regional de Investigación Quilamapu, Casilla 426, \\ Chillán, Chile. \\ *Autor para correspondencia Email: cequezad@udec.cl
}

\section{RESUMEN}

La termografía infrarroja es una metodología eficiente para monitorear el estado hídrico de la planta en condiciones de campo. El objetivo de esta investigación fue validar el uso del Índice de Estrés Hídrico de Cultivo (CWSI por sigla en inglés Crop Water Stress Index) como indicador del estado hídrico de la planta en manzano 'Royal Gala', y evaluar su efecto en rendimiento y calidad, en la Región de Nuble, Chile. El diseño experimental utilizado fue de bloques completamente al azar, con dos tratamientos: con riego y sin riego. Los parámetros evaluados fueron: temperatura de la canopia, humedad del suelo, potencial hídrico xilemático $\left(\Psi_{\mathrm{x}}\right)$, rendimiento y calidad del fruto. Los resultados mostraron diferencias significativas entre tratamientos y una correlación positiva moderada entre potencial hídrico xilemático y CWSI $\left(R^{2}=0,67\right)$. El rendimiento y calidad de la fruta presentó diferencias significativas entre tratamientos; el no regado disminuyó el calibre y rendimiento, pero aumentó la cantidad de sólidos solubles totales. El CWSI no se comportó como un buen predictor del estado hídrico de la planta ni del rendimiento y calidad en manzano.

Palabras clave: potencial hídrico xilemático, manzano, rendimiento, calibre, calidad, cámara térmica, humedad del suelo.

\section{ABSTRACT}

Infrared thermography is an efficient methodology to monitor plant water status under field conditions. The objective of this research was to validate the use of Crop Water Stress Index (CWSI) as an indicator of plant water status in 'Royal Gala' apples and evaluate its effect on yield and fruit quality in the Nuble Region, Chile. The experimental design was completely randomized blocks with two water treatments: irrigated and non-irrigated. Canopy temperature, soil moisture, xylem water potential $(\Psi x)$, fruit yield and fruit quality were evaluated. The results showed significant differences between the treatments and a moderate positive correlation between xylem water potential and CWSI $\left(R^{2}=0.67\right)$. Fruit yield and quality showed significant differences between the treatments; the non-irrigated treatment presented a decrease in size and yield, but an increase in the amount of total soluble solids. The CWSI was not a good indicator of plant water status, fruit yield or fruit quality in 'Royal Gala' apples.

Key words: xilematic hydric potential, apple, yield, fruit size, quality, thermic chamber, soil humidity. 


\section{INTRODUCCIÓN}

La escasez del recurso hídrico en un escenario de cambio climático obliga a aumentar la eficiencia de uso del agua, utilizando métodos de riego localizados y de alta eficiencia, así como realizar programaciones de riego más precisas y diferenciales según el tipo de suelo.

La producción frutícola en Chile ha sido afectada en los últimos años por sequías, con efectos cada vez más intensos en las últimas temporadas agrícolas. Por lo anterior, es de vital importancia monitorear el estado hídrico de las plantas en cada etapa fenológica, para mejorar la programación de riego y obtener mejores rendimientos y calidad de fruta. Los métodos convencionales para estimar el consumo de agua por el sistema suelo - planta se basan en el contenido de humedad de suelo y variables meteorológicas, que proporcionan sólo una información puntual que es compleja de caracterizar.

La evaluación del estado hídrico de las plantas no es una tecnología habitual en los huertos frutales, debido a que los métodos son poco prácticos, y tienen protocolos y procedimientos de tomas de muestras lentos y engorrosos. En la actualidad el más preciso es la determinación del potencial hídrico xilemático $(\Psi \times)$, que diversas investigaciones han demostrado que es el parámetro biofísico más adecuado para establecer niveles de estrés hídrico, ya que integra el déficit de presión de vapor de la atmósfera con la disponibilidad de agua en el suelo explorado por el sistema radical de las plantas (Williams et al., 2012; Bellvert et al., 2015; Alghory y Yazar, 2019; Santesteban et al., 2019). Sin embargo, su determinación es demasiado laboriosa, en cuanto a tiempos de corte y presurización de la hoja, además es una metodología destructiva, pues se requiere la escisión de hojas (Williams et al., 2012).

Otra metodología para estimar las necesidades hídricas de un cultivo es la termografía infrarroja, que monitorea la temperatura de la canopia mediante imágenes infrarrojas usando una cámara térmica. Está técnica no destructiva ha sido validada como un buen indicador del estado hídrico de la planta y detección de estrés hídrico (Fuentes et al., 2012; Khorsandi et al., 2018).

El CWSI es función del diferencial de temperatura canopia-aire y del déficit de presión de vapor (DPV) (Tekelioğlu et al., 2017), siendo un buen indicador del estado hídrico de la planta, ya que la temperatura de la hoja es muy representativa de la variación de la conductancia estomática (Pou et al., 2014), presenta correlación significativa con el potencial hídrico xilemático
(Alghory y Yazar, 2019), y es un promisorio indicador de la disponibilidad de agua en la zona de raíces (Osroosh et al., 2015). Al respecto, Taghvaeian et al. (2012) y Sezen et al. (2014) reportaron que el CWSI puede ser usado para monitorear el estado hídrico, predecir rendimientos y estimar la evapotranspiración del cultivo. Por su parte, Zare et al. (2019) estimaron que el CWSI determinado mediante imágenes satelitales incluye la distribución espacial de este indicador, mediante el mapeo de extensas áreas de huertos de manzanos, lo cual puede ser un método más efectivo de monitoreo del estrés hídrico y programación de riego.

Por lo anteriormente expuesto, el objetivo de esta investigación fue validar el uso del CWSI, como indicador del estado hídrico de la planta, $y$ su efecto en el rendimiento y calidad en manzanos 'Royal Gala'

\section{MATERIALES Y MÉTODOS}

\section{Sitio experimental}

El ensayo de campo se realizó en el predio Santa Rosa del Centro Regional de Investigación Quilamapu, del Instituto de Investigaciones Agropecuarias (INIA), ubicado en el $\mathrm{km} 22$ camino a Cato (36 $32^{\prime} 16^{\prime \prime}$ S; $71^{\circ} 55^{\prime} 21^{\prime \prime}$ O; 191 m.s.n.m.). El huerto experimental tiene una superficie de 3.000 $\mathrm{m}^{2} \mathrm{y}$ está plantado con los cultivares Royal Gala y Fuji, en un marco de plantación de $3 \mathrm{~m}$ entre hilera y $1 \mathrm{~m}$ sobre hilera. El suelo es un Andisol, serie Arrayán, clasificado como Medial, amorphic, thermic humic Haploxerands, con profundidad superior a $90 \mathrm{~cm}$, de topografía plana, la textura es franco arcillo limosa a franco limosa en superficie y franca a franco arenosa en profundidad, drenaje moderado a bueno (Stolpe, 2006). El clima es de tipo Mediterráneo perteneciente al agroclima Santa Rosa. Las precipitaciones anuales varían entre 1.000 a $1.200 \mathrm{~mm}$ y la evapotranspiración potencial entre 860 y $1.000 \mathrm{~mm}$. La temperatura media anual es de $13,6^{\circ} \mathrm{C}$, el periodo libre de heladas dura 5 meses y la humedad relativa anual promedio es del $67 \%$. El mayor déficit hídrico se presenta entre los meses de octubre-marzo (del Pozo y del Canto, 1999).

\section{Riego}

El riego se aplicó por goteo, con emisores integrales autocompensados de $8 \mathrm{~L} \mathrm{~h}^{-1}$ (Cosmoplas, modelo Corona, marca Eurodrip, Atenas, Grecia), 1 gotero por árbol, con presión de operación de 10 mca. El diseño contempla un lateral de polietileno (PE) por hilera con una longitud de $160 \mathrm{~m}$. La programación de riego fue variable en función de la características del suelo y evapotranspiración del cultivo, con frecuencia 
de riego entre 4 a 6 días y tiempo de riego entre 3 a 6 horas.

\section{Diseño experimental}

El diseño experimental utilizado fue de bloques completamente al azar, con dos tratamientos hídricos: $\mathrm{T}_{1}$ : Riego por goteo con emisores de $8 \mathrm{~L} \mathrm{~h}^{-1}$, y $\mathrm{T}_{2}$ : Sin riego, desde el 16 de diciembre del 2015, hasta término de cosecha el 25 de febrero del 2016. El diseño consideró 3 repeticiones por tratamiento, dos con 3 árboles y una con 4 árboles, con un total de 10 árboles por tratamiento. Para evitar el efecto borde en los tratamientos con estrés hídrico, se dejó una hilera de seguridad a cada lado.

\section{Evaluaciones}

Se registraron 960 datos, correspondientes a temperaturas mínimas, medias y máximas de la canopia del manzano, potencial hídrico xilemático $(\Psi x)$ y del suelo, los cuales se graficaron para evaluar la variación a través del tiempo. Además, se aplicaron regresiones lineales para determinar la relación de dependencia entre variable dependiente e independientes. Para rendimiento y calidad, se recopilaron 820 datos que se subdividieron en 2 tratamientos de 10 árboles regados y 10 árboles no regados.

\section{Potencial hídrico xilemático}

La determinación del $\Psi x$ se realizó con una cámara de presión tipo Scholander (marca Solfranc, modelo Sf-Pres-35, Tarragona, España), las hojas seleccionadas eran de tamaño medionormal $(10-15 \mathrm{~cm})$, sanas, sin deformaciones ni daños, de color verde y peciolo lignificado, ubicadas en la zona central del dosel del árbol. Las hojas se cubrieron durante $2 \mathrm{~h}$ antes de la medición con una bolsa plástica y papel aluminio. Se seleccionaron 2 hojas de la zona superior y 2 hojas de la zona basal, de cada árbol de cada tratamiento, obteniéndose cuatro datos, los cuales fueron promediados, dando en total 80 datos por cada tratamiento. Las mediciones se realizaron entre el 16-12-2014 y 11-02-2015, es decir durante los meses de mayor déficit de presión de vapor, con una frecuencia variable entre 6 a 8 días. $\mathrm{Al}$ momento de la medición, las hojas seleccionadas fueron cortadas del árbol y colocadas en la cámara hermética con el peciolo hacia afuera; el tiempo transcurrido entre el corte de la hoja y la introducción a la cámara no superó 1 min. Luego se selló con un tapón de goma, y se abrió la llave del gas (nitrógeno a presión) incrementando la presión cada 0,2 bar hasta el momento que apareció la primera gota en el peciolo. Las mediciones fueron obtenidas entre las 12:40 y 14:30 h.

\section{Temperatura de la canopia}

La temperatura de la canopia se midió entre las 12:00 y 15:00 h en días despejados; utilizando una cámara térmica (Fluke, Ti55FT-20, Everett, USA), con una sensibilidad de $0,05^{\circ} \mathrm{C}$ y un rango espectral de $8 \mu$ a $14 \mu \mathrm{m}$. La captura de imágenes fue en dirección oeste-este, a una distancia de 60 $\mathrm{cm}$ del follaje y en la zona media del árbol, con un ángulo de $45^{\circ}$, asegurando que todo el campo de visión del aparato estuviera cubierto por la superficie foliar, evitando objetos ajenos a la medición. Posteriormente, las imágenes fueron procesadas con la cámara Fluke asociada al uso del software SmartView y luego se exportaron a formato texto (txt). Este proceso permite visualizar y procesar las imágenes capturadas, generando un archivo txt el cual entrega la totalidad de las temperaturas presentes en cada imagen de $320 \times 240$, pixel por pixel, excluyendo todos los objetos ajenos a la canopia del manzano, que producen distorsión en ella, tales como suelo, ramas principales, y cielo. Una vez filtradas las imágenes, se obtuvieron las temperatura mínima, media y promedio, obteniendo un total parcial de 30 datos por cada fecha de medición, siendo 8 fechas diferentes resultando un total final de 240 datos por cada tratamiento y un total de 480 datos para el estudio.

El CWSI fue determinado mediante la siguiente relación empírica (Jones, 1999):

CWSI $=($ Tc-Tmín $) /($ Tmáx - Tmín $)$

donde: $\mathrm{Tc}=$ temperatura media de canopia; Tmín = temperatura mínima de canopia para el cultivo no estresado; Tmáx = temperatura máxima de canopia para el cultivo estresado.

\section{Humedad de suelo}

Las mediciones del contenido de humedad del suelo fueron realizadas simultáneamente en los días de medición de temperatura de canopia y potencial hídrico xilemático. El muestreo de suelos fue realizado en cada árbol seleccionado por tratamiento, y fueron extraídas a una profundidad de $60 \mathrm{~cm}$ en la zona radical y a $20 \mathrm{~cm}$ del emisor más cercano a la planta. Las muestras se registraron por fecha, número de árbol y tratamiento. Luego el suelo fue pesado y secado en un horno a $105^{\circ} \mathrm{C}$ durante $48 \mathrm{~h}$ hasta obtener peso constante, obteniendo la humedad base suelo seco (\% hbss) mediante el método gravimétrico (Sandoval et al., 2012).

\section{Cosecha}

El momento óptimo de cosecha se definió en función de los días después de plena flor (DDPF), que se considera la fecha en la cual el $50 \%$ de las flores están abiertas y son constantes entre localidades y temporada. En base a este criterio 
la cosecha se realizó el día 25 de febrero del 2015, ya que la floración fue homogénea por la alta densidad de plantación.

\section{Sólidos Solubles Totales (SST)}

La concentración de sacarosa se midió en 10 frutos por cada árbol seleccionado de cada tratamiento, obteniendo un total de 200 datos. Con este fin se cortó un pedazo de fruta, se ejerció presión manual, extrayendo el jugo de la pulpa, que se esparció en la zona anterior y se midió por el visor que se encontraba en la zona posterior. Se utilizó un refractómetro óptico (ATAGO, modelo PAL-84S, Tokio, Japón).

\section{Calibre}

La medición del diámetro ecuatorial $(\mathrm{mm})$ en la zona media del fruto, se determinó en 10 frutos por cada árbol de cada tratamiento, obteniendo un total de 200 datos de calibre (mm), utilizando un pie de metro (Mitutoyo, 500172, Kawasaki, Japón).

\section{Color}

Se realizó una estimación visual del porcentaje de cubrimiento de color de la fruta, en 10 frutas por cada árbol muestreado por cada tratamiento, obteniendo un total de 200 datos. Esta estimación varió entre $0-100 \%(0=$ sin color; $100=$ color rojo completo).

\section{Rendimiento}

El rendimiento se obtuvo cosechando el total de frutos de 10 árboles correspondiente a cada tratamiento, es decir 20 árboles que contemplaba el ensayo. Los rendimientos se calcularon según el peso promedio total $(\mathrm{kg})$ de los 10 árboles de cada tratamiento, multiplicados por la densidad de plantación (3.333 plantas ha ${ }^{-1}$ ). Se utilizó una balanza digital de $30 \mathrm{~kg}$ (Maigas, DY778B, Santiago, Chile).

\section{Análisis estadístico}

Los resultados obtenidos fueron sometidos a un análisis de varianza (ANDEVA) de un factor, y las diferencias significativas $(P<0,05)$, entre las medias de los tratamientos se analizaron con el test de Duncan. La normalidad de datos se verificó con el test de Shapiro-Wilks. Además, se realizaron correlaciones de datos mediante regresión lineal con el programa estadístico Infostat (Balzarini et al., 2008)

\section{RESULTADOS Y DISCUSIÓN}

\section{Potencial hídrico xilemático}

El efecto de los tratamientos hídricos en el potencial hídrico xilemático (Fig. 1) permite observar diferencias entre sin riego, con valores entre -11 y -14 bar, y regado, con valores entre

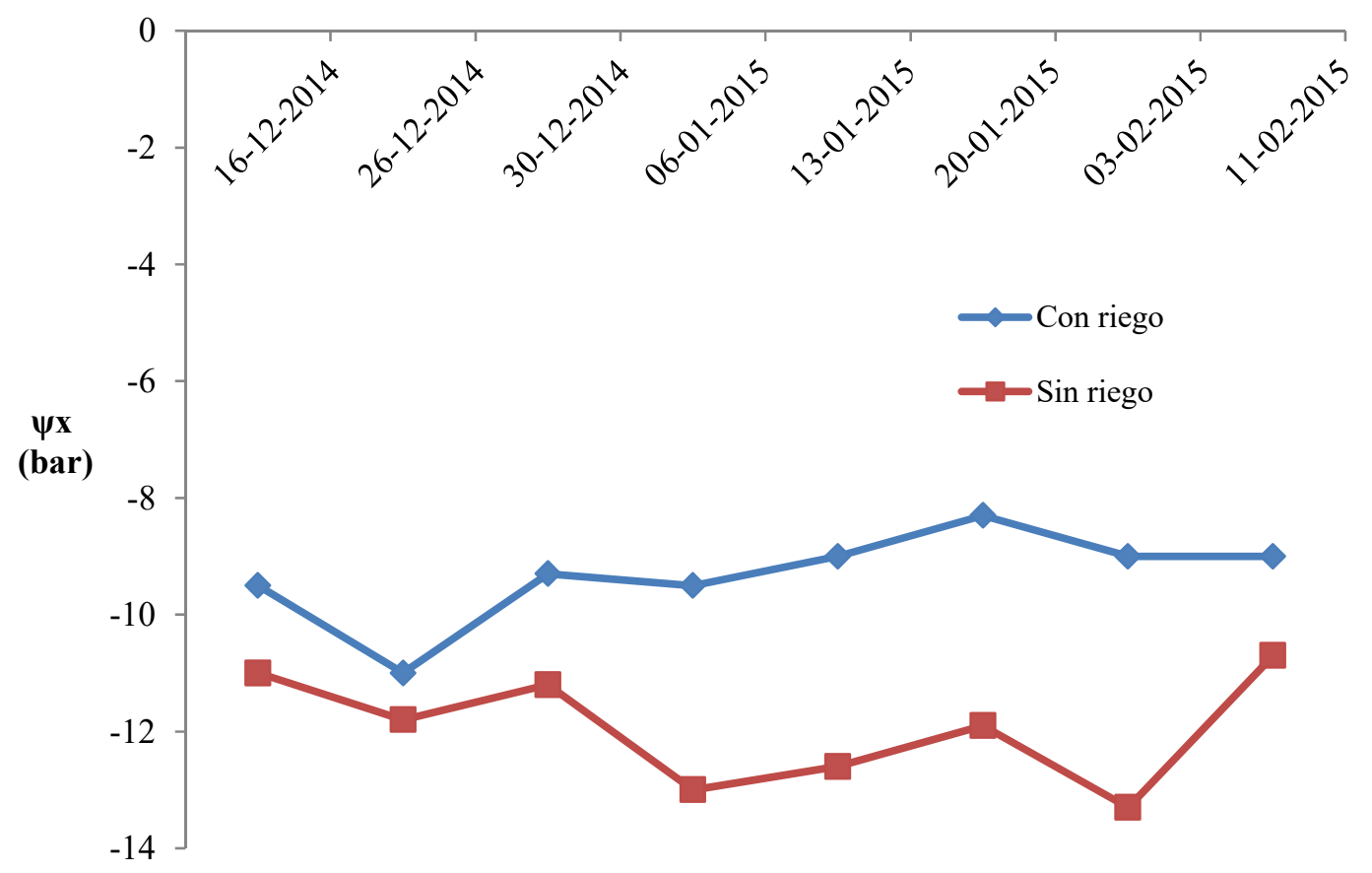

Fig. 1. Potencial hídrico xilemático con dos tratamientos hídricos en manzanos ‘Royal Gala' en un suelo Haploxerands en la Región de Ñuble, Chile.

Fig. 1. Xylem water potential in 'Royal Gala' apple trees under two water treatments in a Haploxerands soil, Nuble Region, Chile. 
-9 y -10 bar. Esto resultados concuerdan con lo reportado por Chenafi et al. (2016) quienes establecieron niveles críticos entre -12 a -14 bar en manzanos 'Fuji'. Una diferencia más amplia fue determinada entre la medición del 06 de enero del 2015 y 03 de febrero del 2015, debido a que en ese periodo el manzano inicia su etapa de división y elongación celular. Por ende, este periodo es el de mayor requerimiento hídrico y el de mayor déficit de presión de vapor, por lo que se hace más significativo el efecto de la escasez hídrica en la planta.

El tratamiento sin riego presentó los valores de $\Psi x$ más bajos, entre -11 y -14 bar, especialmente en el mes de febrero, y con mayor grado de estrés, pero con diferencias moderadas en el mes de diciembre con respecto al tratamiento con riego, debido a la alta capacidad de retención de agua de los suelos serie Arrayán (Haploxerands), y a la profundidad del suelo, mayor a $90 \mathrm{~cm}$, lo que permite una mayor exploración del sistema radical para suplir los requerimientos hídricos del árbol, para el desarrollo y maduración de la fruta.

\section{Índice de estrés hídrico del cultivo (CWSI)}

El CWSI varió en función de los tratamientos hídricos (Fig. 2). Con riego presentó menores valores, siendo 0,19 el más bajo y 0,43 el más alto, en comparación al no regado, con valores de 0,35 el más bajo y 0,91 el más alto, durante la temporada. Si bien los valores de CWSI son diferentes, no concuerdan con lo determinado en vid vinífera por Bellvert et al. (2015) quienes reportaron que un tratamiento bien regado tiene CWSI menor a 0,3 , lo que puede estar asociado al estado fenológico, a la disponibilidad de agua y a la demanda por evapotranspiración.

$\mathrm{Al}$ respecto, Nagy et al. (2015) expresaron que el uso de imágenes térmicas y la determinación del CWSI pueden ser un indicador previo a la aparición de síntomas visibles de estrés hídrico, facilitando el manejo del riego por goteo en los huertos de manzanos, concluyendo que si el CWSI es mayor a 0,3 disminuye la tasa de crecimiento, siendo el rango de 0,2 a 0,3 representativo de un buen estado hídrico del frutal, lo que en esta investigación se logró entre el 20 de enero y el 10 de febrero de 2015. Sin embargo, antes del 20 de enero el CWSI obtenido estuvo entre 0,4 y 0,6 debido a que el sistema no cumplió con los requerimientos hídricos del cultivo, por problemas de mantención, uniformidad de caudales, y desuniformidad en las dimensiones de los bulbos de mojamiento.

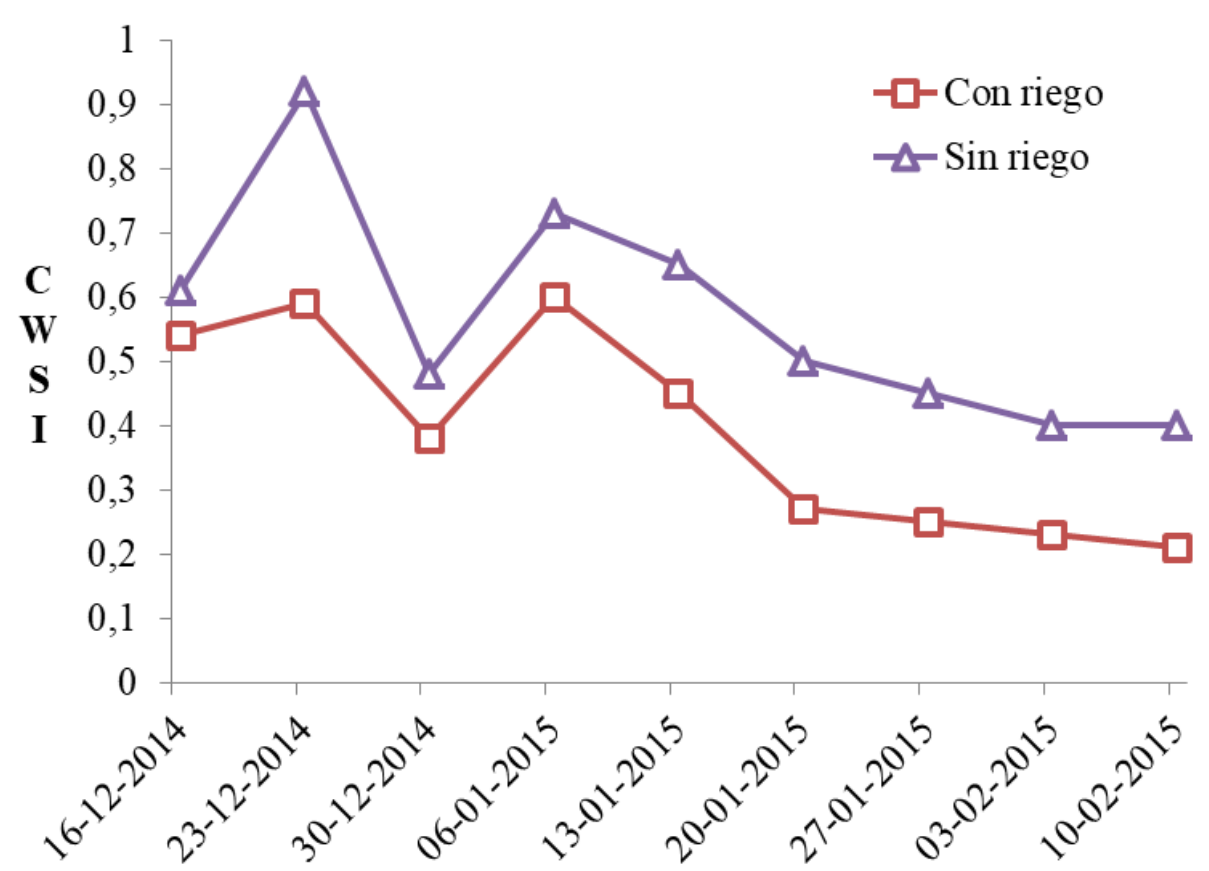

Fig. 2. CWSI en manzanos 'Royal Gala' con dos tratamientos hídricos en un suelo Haploxerands en la Región de Nuble,Chile. $T_{1}$ : Con riego; $T_{2}$ : Sin riego.

Fig. 2. CWSI in 'Royal Gala' apple trees under two different water treatments in a Haploxerands soil, Nuble Region, Chile. $T_{1}$ : Irrigated; $T_{2}$ : Non- irrigated 
Relación entre el potencial hídrico xilemático y CWSI

La relación entre $\Psi x$ y CWSI (Fig. 3), presentó un $\mathrm{R}^{2}=0,77$ que es considerado una correlación media, y similar a la obtenida por Wang y Carting (2010) en duraznos, lo que significa que no es un predictor exacto del estado hídrico de la planta. Si bien la calidad del modelo es medianamente baja, esto puede ser explicado por la influencia de la humedad relativa, el viento y otras variables ambientales, que al momento de la captura de la imagen térmica para la obtención de CWSI pueden disminuir o aumentar la temperatura del árbol, causando un error en la toma de datos de temperatura realizados por la cámara térmica. Sin embargo, Osroosh et al. (2015), al correlacionar CWSI y $\Psi x$ en manzanos, obtuvieron un $\mathrm{R}^{2}=$ 0,91 , debido a que realizaron modificaciones de CWSI que eliminaban el grado de error que provocaban factores externos en la toma de muestras de temperaturas de la canopia, como viento y humedad relativa. Sin embargo, Bellvert et al. (2015) determinaron que la correlación entre CWSI y potencial de la hoja en vid vinífera debe ser calibrada en función de variedad y estado fenológico, ya que encontraron $R^{2}=0,634$ y $R^{2}=$ 0,729 , respectivamente, lo que también podría ser aplicable en manzanos.

\section{Relación entre humedad del suelo y CWSI}

La relación de humedad de suelo y CWSI (Fig. 4) presentó una baja correlación $\left(R^{2}=0,41\right)$ mostrando una tendencia a la disminución del CWSI al aumentar la humedad de suelo, no siendo un parámetro adecuado para determinar disponibilidad de agua en la zona de raíces. Sin embargo, Taghvaeian et al. (2012) determinaron un alto coeficiente de determinación $\left(\mathrm{R}^{2}=0,89\right)$ entre CWSI y la humedad en volumen, pero esta se determinó en el horizonte superficial entre $10 \mathrm{y} 14 \mathrm{~cm}$. En este sentido, Osroosh et al. (2015) determinaron una alta sensibilidad del CWSI promedio durante las horas de luz del día con la disponibilidad de agua en la zona de raíces $\left(R^{2}\right.$ $=0,70)$. Por su parte, Han et al. (2018) proponen que el CWSI se puede utilizar para mejorar la estimación de la disponibilidad agua en la zona de raíces y precisar el método del balance hídrico.

\section{Relación CWSI y rendimiento}

El índice de estrés hídrico del cultivo (CWSI) promedio vs rendimiento presentó un $\mathrm{R}^{2}=0,56$ (Fig. 5) lo que refleja que el CWSI no es un buen predictor del rendimiento. La disminución del rendimiento con el aumento del CWSI se debe a que el árbol estresado utiliza sus reservas de agua y energía en subsistir y revertir ese estado, no en proporcionar esos elementos a la producción de fruta.

Esta baja influencia de la temperatura como predictor se puede explicar porque hay otros factores que afectan el rendimiento, como el manejo del agua, el manejo de la carga frutal, raleo de flores, podas, y fertilización. Además, la toma de muestras puede causar distorsiones debido a la velocidad del viento, humedad relativa o toma de imágenes mal realizadas, que causan temperaturas erróneas para la formulación del CWSI. Al respecto, O'Shaughnessy et al. (2011) relacionaron el CWSI modificado con algoritmos (CWSIe) con

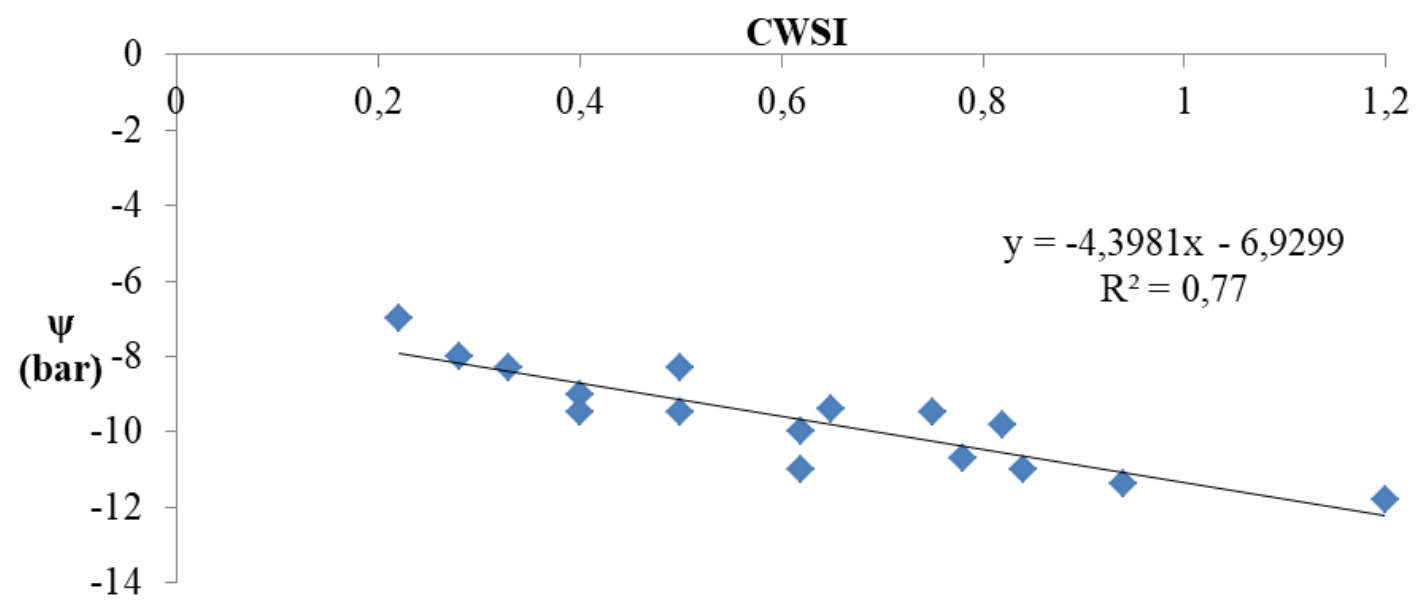

Fig. 3. Regresión lineal entre $\Psi$ x y CWSI en manzanos ‘Royal Gala' con dos tratamientos hídricos en un suelo Haploxerands en la Región de Nuble, Chile.

Fig. 3. Linear regression between $\Psi x$ and CWSI in 'Royal Gala' apple trees under two different water treatments in a Haploxerands soil, Nuble Region, Chile. 


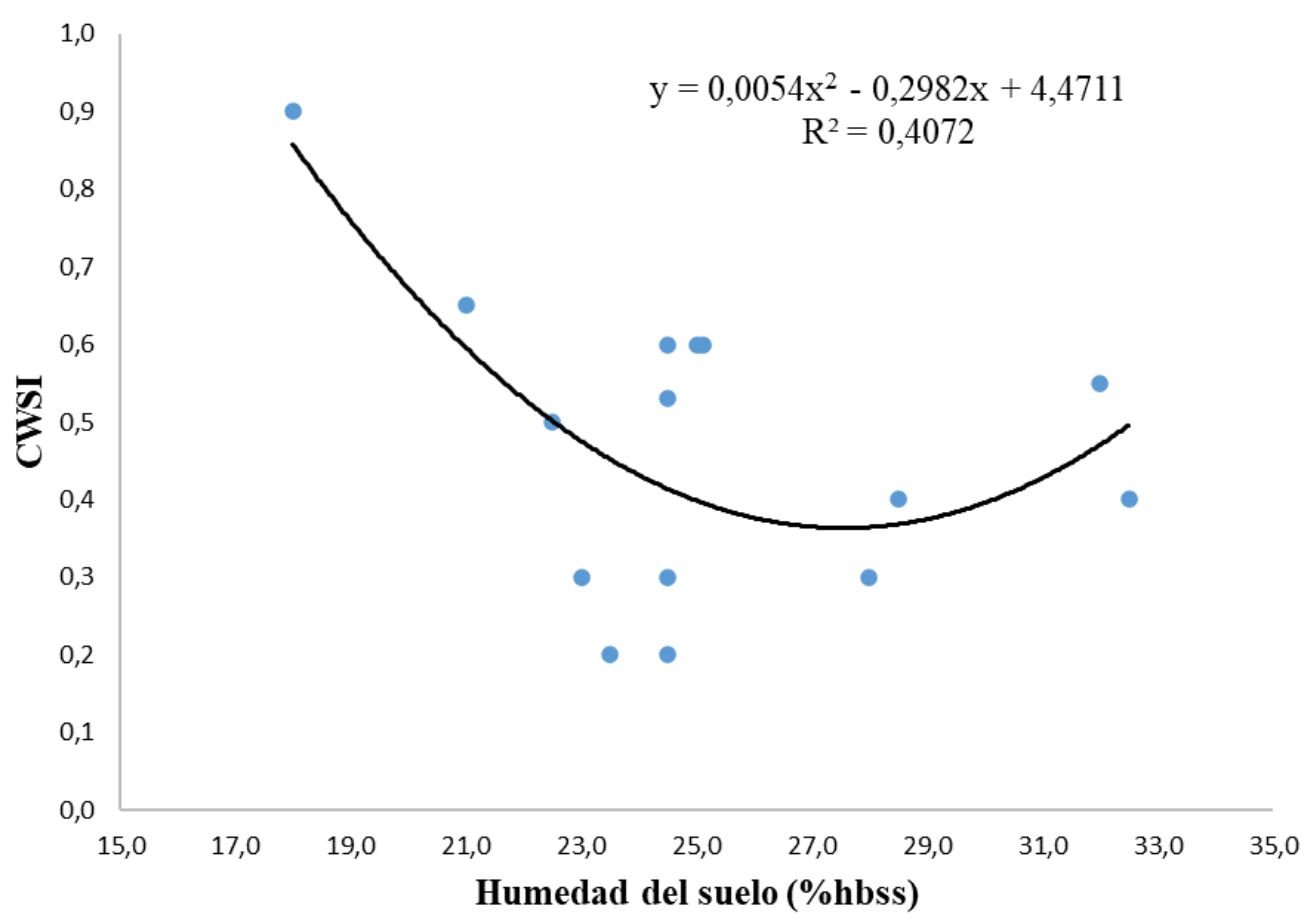

Fig. 4. Regresión lineal entre humedad de suelo (\%hbss) y CWSI en manzanos 'Royal Gala' con dos tratamientos hídricos en un suelo Haploxerands en la Región de Ñuble, Chile.

Fig. 4. Linear regression between soil moisture and CWSI in 'Royal Gala' apple trees under two different water treatments in a Haploxerands soil, Nuble Region, Chile.

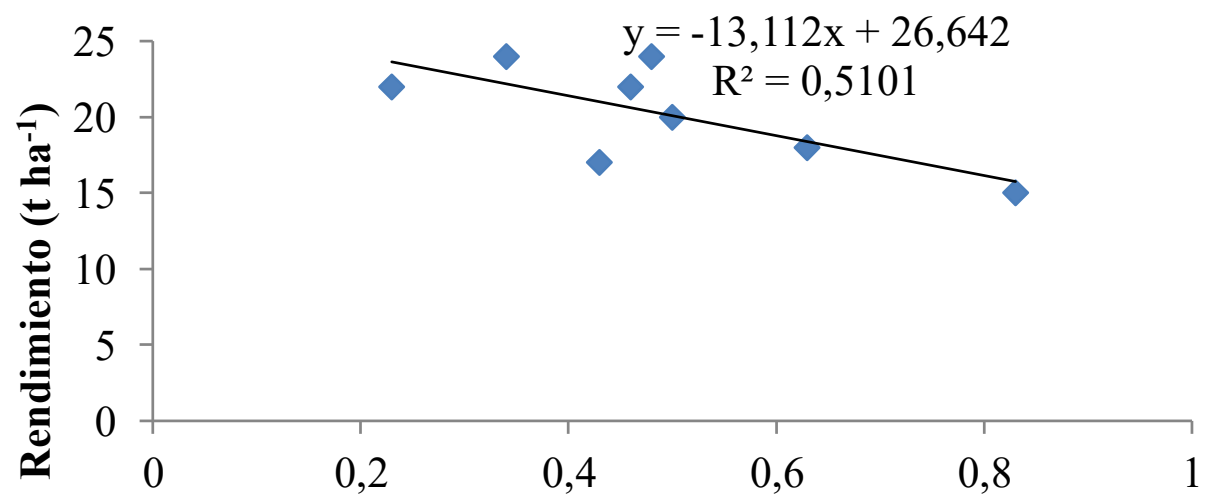

CWSI (0-1)

Fig. 5. Regresión lineal entre CWSI y rendimiento en manzanos 'Royal Gala' con dos tratamientos hídricos en un suelo Haploxerands en la Región de Ñuble, Chile.

Fig. 5. Linear regression between CWSI and yield in 'Royal Gala' apple trees under two different water treatments in a Haploxerands soil, Ñuble Region, Chile. 
rendimiento en algodoneros, obteniendo un $\mathrm{R}^{2}$ $=0,88$, concluyendo que con menores CWSI se obtuvieron los mayores rendimientos.

\section{Rendimiento y calidad}

Los parámetros de rendimiento y calidad presentaron diferencias significativas $(P<0,05)$ en función de los tratamientos hídricos (Tabla 1). En el tratamiento regado se obtuvo un rendimiento de $27,7 \mathrm{t} \mathrm{ha} \mathrm{a}^{-1}$ en comparación a $18,1 \mathrm{t} \mathrm{ha}^{-1}$ en el tratamiento sin riego, lo que representa una disminución de rendimiento del $34,6 \%$, pero menor a la determinada por Girona et al. (2010) en manzanos 'Golden Smoothee', con diferencias de hasta $50 \%$ de rendimiento entre riego semanal y los tratamientos de déficit hídrico. Al respecto, Zhong et al. (2019) obtuvieron rendimientos de $45 \mathrm{t} \mathrm{ha}^{-1}$ con riego y $25 \mathrm{t} \mathrm{ha}^{-1}$ sin riego, lo que representa una disminución del $44,4 \%$. Esto puede ser explicado porque el déficit hídrico afecta la división y elongación celular produciendo una disminución significativa del rendimiento en manzanas, especialmente en la etapa III de crecimiento del fruto.

El calibre del fruto fue mayor en el tratamiento regado, con un promedio de $68,3 \mathrm{~mm}$ de diámetro en relación a $66,09 \mathrm{~mm}$ del no regado, por el agotamiento de las reservas hídricas, ya que al estar en periodo de elongación y división celular no dispone del agua suficiente para generar frutas de mayor tamaño, así también hay una disminución de la fotosíntesis debido al cierre de estomas, lo que origina menor crecimiento de frutos. Esto concuerda con Quezada et al. (2011) quienes obtuvieron mayores diámetros de fruto con incremento de la reposición hídrica entre 50 y $100 \%$ de la evapotranspiración del cultivo (ETc) con riego por goteo.

Los parámetros de calidad presentaron diferencias significativas entre tratamientos $(\mathrm{P}<$ 0,05), debido a las diferentes condiciones hídricas de las plantas, ya que los periodos fenológicos más sensibles al déficit hídrico corresponden a la etapa II (floración y fructificación) y etapa III (crecimiento del fruto) (Zhong et al., 2019).

En relación al porcentaje de cubrimiento de color, el tratamiento con riego obtuvo el mayor valor con 93,67\%, debido al mayor desarrollo de antocianinas, ya que según Zhong et al. (2019) el déficit hídrico no tiene efectos significativos en el grado de color. Esto puede explicarse porque el desarrollo del color está asociado a la radiación solar, y los árboles regados tuvieron mayor captación de luz que los árboles sin riego, debido a una irregular luminosidad en el huerto asociado a posición de los árboles y manejo de la poda. Esto concuerda con Umanzor et al. (2017) quienes determinaron que la radiación fotosintéticamente activa total y difusa incrementaron el desarrollo del color y la síntesis de antocianinas en manzanos 'Fuji' y 'Gala'.

Los resultados de SST fueron mayores en el tratamiento sin riego con $16,86^{\circ}$ Brix en comparación a $14,15^{\circ}$ Brix del tratamiento regado, lo que concuerda con Chenafi et al. (2016) quienes determinaron una leve tendencia a una mayor concentración de sólidos solubles con el aumento del estrés hídrico, lo que estaría asociado a un efecto de concentración por el menor tamaño del fruto. Similares resultados fueron obtenidos en manzanos por Zhong et al. (2019), quienes determinaron que un déficit hídrico entre floración y fructificación mejoró el contenido de azúcares y sólidos solubles y tuvo un efecto positivo en la calidad de la fruta.

\section{CONCLUSIONES}

La relación entre CWSI y $\Psi x$ con un $\mathrm{R}^{2}=0,68$ indica que es un predictor poco preciso del estado hídrico de la planta. El CWSI no se comportó como un buen predictor del rendimiento y calidad en manzanos 'Roya Gala' y presentó una baja correlación con la humedad del suelo en la zona de raíces. Los tratamientos con estrés hídrico en manzano disminuyeron el calibre y el rendimiento pero aumentaron los sólidos solubles totales.

Tabla 1. Rendimiento y parámetros de calidad, en manzanos 'Royal Gala' con dos tratamientos hídricos en un suelo Haploxerands en la Región de Nuble, Chile.

Table 1. Fruit yield and quality in 'Royal Gala' apple trees under two different water treatments in a Haploxerands soil, Nuble Region, Chile.

\begin{tabular}{ccccc}
\hline Tratamiento & $\begin{array}{c}\text { Rend. } \\
\left(\mathbf{t ~ h a}^{-1} \mathbf{)}\right.\end{array}$ & $\begin{array}{c}\text { Calibre } \\
\mathbf{( m m )}\end{array}$ & $\begin{array}{c}\text { Color } \\
\mathbf{( \% )}\end{array}$ & $\begin{array}{c}\text { SST } \\
\left.\mathbf{(}^{\circ} \text { Brix }\right)\end{array}$ \\
\hline $\mathrm{T} 1$ & $27,7 \mathrm{a}$ & $68,30 \mathrm{a}$ & $93,67 \mathrm{a}$ & $14,50 \mathrm{~b}$ \\
$\mathrm{~T} 2$ & $18,1 \mathrm{~b}$ & $66,09 \mathrm{~b}$ & $88,67 \mathrm{~b}$ & $16,86 \mathrm{~b}$ \\
\hline
\end{tabular}

T1: Con riego; T2: Sin riego. 


\section{LITERATURA CITADA}

Alghory, A., and A. Yazar. 2019. Evaluation of crop water stress index and leaf water potential for deficit irrigation management of sprinkler-irrigated wheat. Irrig. Sci. 37(1):6177.

Balzarini, M., A.Di Rienzo, F. Cazanoves, L. González, M. Tablada, W. Guzmán, et al. 2008. InfoStat software estadístico. InfoStat versión 2008, Manual de usuario. Grupo InfoStat, FCA, Universidad Nacional de Córdoba, Argentina.

Bellvert, J., J. Marsal, J., J. Girona, and P.J. ZarcoTejeda. 2015. Seasonal evolution of crop water stress index in grapevine varieties determined with high-resolution remote sensing termal imagery. Irrig. Sci. 33(2):8193.

Chenafi, A., P. Monney, E. Arrigoni, A. Boudoukha, and C. Carlen. 2016. Influence of irrigation strategies on productivity, fruit quality and soil-plant water status of subsurface drip-irrigated apple trees. Fruits 71(2):69-78.

del Pozo, A., y P. del Canto. 1999. Áreas agroclimáticas y sistemas productivos en la VII y VIII regiones. Serie Quilamapu № 113. INIA Quilamapu, Chillán, Chile.

Fuentes, S., R. De Bei, J. Pech, and S. Tyerman. 2012. Computational water stress indices obtained from thermal image analysis of grapevine canopies. Irrig. Sci. 30(6):523-53.

Girona, J., M.H. Behboudian, M. Mata, J, Del Campo, and J. Marsal. 2010. Exploring six reduced irrigation option under water shortage for 'Golden Smoothee' apple: Responses of yield components over three years. Agric. Water Manage. 98(2):370-375.

Jones, H.G. 1999. Use of thermography for quantitative studies of spatial and variation of stomatal conductance over leaf surfaces. Plant, Cell \& Environment 22:1043-1055.

Khorsandi, A., A. Hemmet, S. Ahmad Mireei, R. Amirfattahi, and P. Ehsanzadeh. 2018. Plant temperature-based indices using infrared thermography for detecting water status in sesame under greenhouse conditions. Agric. Water Manage. 25(1):222-233.

Han, M., H. Zhang, J.L. Chávez, L. Ma, and T.J. Trout. 2018. Improved soil water deficit estimation through the integration of canopy temperature measurements into a soil water balance model. Irrig. Sci. 36(3):187-201.

Nagy, A. 2015. Thermographic evaluation of water stress in an apple orchard. J. of Multi. Eng. Sci. Tech. 2(8):2210-2215.
O'Shaughnessy, S., S. Evett, P. Colaizzi, and T. Howeell. 2011. Using radiation thermography and thermometry to evaluate crop water stress in soybean and cotton. Agric. Water Manage. 98(10):1523-1535.

Osroosh, Y., T. Peters, C.S. Campbell, and Q. Zhang. 2015. Automatic irrigation scheduling of apple threes using theorical crop water stress index with an innovate dynamic threshold. Comp. Elect. Agric. 118:193-203.

Pou, A., M.P. Diago, H. Medrano, J. Baluja, and J. Tardaguila. 2014. Validation of thermal indices from water status identification in grapevine. Agric. Water Manage. 134:60-72.

Quezada, C., E. Solís, A. Venegas, y M. Faúndez. 2011. Efecto de cuatro niveles de aplicación de agua en rendimiento y calidad de un huerto de manzano (Malus domestica Bork.) 'Fuji ' bajo riego por goteo. Agrociencia 27(2):65-75.

Sandoval., M., J. Dörner, O. Seguel, J. Cuevas, y D. Rivera. 2012. Métodos de análisis físico de suelos. 80 p. Universidad de Concepción. Publicaciones de Suelos y Recursos Naturales № 5, Chillán, Chile.

Santesteban, L.G., C. Miranda, D. Marín, B. Sesma, D.S. Intrigliolo, J.M. Mirás-Avalos, et al. 2019. Discrimination ability of leaf and stem water potential at different times of the table day through a meta-analysis in grapevine (Vitis vinifera L.). Agric. Water Manage. 221:202210.

Sezen, S.M., A. Yazar, H.Y. Dasgan, S. Yucel, A. Akyldiz, S. Tekin, et al. 2014. Evaluation of crop water stress index (CWSI) for red pepper with drip and furrow irrigation under varying irrigation regime. Agric. Water Manage. 143:59-70.

Stolpe, N.B. 2006. Descripción de los principales suelos de la VIII Región de Chile. Universidad de Concepción, Facultad de Agronomía, Chillán, Chile.

Taghvaeian, S., J.L. Chávez, and N.C. Hansen. 2012. Infrared thermometry to estimate crop water stress index and water use of irrigated maize in Northeastern Colorado. Remote Sens. 4:3619-3637.

Tekelioğlu, B., D. Büyüktaş, R. Baştuğ, C. Karaca, K, Aydinşakir, and N. Dinç. 2017. Use of Crop Water Stress Index for irrigation scheduling of soybean in Mediterranean conditions. Journal of Experimental Agriculture International 18(6):1-8.

Umanzor,C., R. Bastías, R. Wilckens, and C. Quezada. 2017. Influence of using of Pearl and red nets on micro-climate conditions, fruit sun damage and fruit color development in 'Gala' and 'Fuji' apple orchards. Rev. Bras. Frutic. 39(1):1-13. 
Wang, D., and J. Carting. 2010. Infrared canopy temperature of early-ripening peach trees under postharvest deficit irrigation. Agric. Water Manage. 97:1787-1794.

Williams, L.E., P. Baeza, and P. Vaughn. 2012. Midday measurements of leaf water potential and stomatal conductance are highly correlated with daily water use of Thompson Seedless. Irrig, Sci. 30(3):201-212.

Zare, M., K, Drastig, and M. Zude-Sasse. 2019. Estimating tree water status in apple orchard using reflectance in the thermal domain of Landsat 8 Satellite. p. 255-259. IEEE International Workshop on Meteorology for Agriculture and Forestry (MetroAgriFor), Portici, Italy.
Zhong, Y., L. Fei, Y. Li, J. Zeng, and Z. Dai. 2019. Response of fruit yield, fruit quality, and water use efficiency to water deficits for apple trees under surge-root irrigation in the Loess Plateau of China. Agric. Water Manage. 222:221-300. 